\title{
Fatores Associados ao Atraso entre o Diagnóstico e o Início do Tratamento de Câncer de Mama: um Estudo de Coorte com 204.130 Casos no Brasil
}

doi: https://doi.org/10.32635/2176-9745.RBC.2020v66n3.979

\author{
Factors Associated with Delay between Diagnosis and Initiation of Breast Cancer Treatment: a Cohort Study with 204.130 \\ Cases in Brazil \\ Factores Asociados con el Retardo entre el Diagnóstico y el Inicio del Tratamiento del Cáncer de Mama: un Estudio de \\ Cohorte con 204.130 Casos en Brasil
}

\begin{abstract}
Giselle Coutinho de Medeiros'; Clarice Gomes Chagas Teodózio²; Erica Alves Nogueira Fabro³; Suzana Sales de Aguiar"; Artur Henrique Machado Lopes ${ }^{5}$; Bárbara Cordeiro de Conte ${ }^{6}$; Erisvan Vieira da Silva7; Lyssandra Luiza Pestana Coelho ; Nitza Ferreira Muniz ${ }^{9}$; Sara Isabel Pimentel de Carvalho Schuab ${ }^{10}$; Anke Bergmann ${ }^{11}$; Luiz Claudio Santos Thuler ${ }^{12}$
\end{abstract}

\section{RESUMO}

Introduçáo: $\mathrm{O}$ câncer de mama é considerado um problema de saúde pública, tendo crescente incidência mundial. Diversos fatores contribuem para o diagnóstico tardio e dificultam o início do tratamento, repercutindo em um pior prognóstico. Objetivos: Analisar o intervalo de tempo entre o diagnóstico e o início do primeiro tratamento oncológico na populaçáo brasileira, além de avaliar os fatores associados aos maiores intervalos. Método: Trata-se de um estudo de coorte retrospectivo com 540.529 pacientes cadastrados no Sistema de Registros Hospitalares de Câncer (SisRHC) no período de 2000 a 2017. Utilizou-se como desfecho o intervalo de tempo entre o diagnóstico e o início do primeiro tratamento oncológico, considerando-se como atraso o intervalo maior do que 60 dias. Para análise das variáveis, foram realizadas análise descritiva e regressão logística simples (IC95\%; p<0,05). Resultados: Foram analisados 204.130 casos que apresentaram média de idade de 55,8 anos $( \pm 13,24)$, sendo predominantemente do sexo feminino (99,1\%), 55,1\% eram da Região Sudeste e 71,4\% residiam em cidades não capitais. A mediana do intervalo de tempo entre o diagnóstico e o início do primeiro tratamento oncológico foi de 63 dias (variação interquartil = 36-109). As variáveis sociodemográficas, clínicas e relacionadas ao tratamento mostraram impacto no intervalo de tempo, com exceçáo da variável sexo. Conclusáo: $\mathrm{O}$ tempo entre o diagnóstico e o início do primeiro tratamento oncológico foi elevado. Fatores sociodemográficos, clínicos e relacionados ao tratamento influenciam nos intervalos de tempo. Identificá-los precocemente pode contribuir para o direcionamento de açóes a esses grupos mais vulneráveis ao atraso.

Palavras-chave: Neoplasias da Mama/diagnóstico; Tempo para o Tratamento; Fatores de Risco; Registros Hospitalares.

\section{ABSTRACT}

Introduction: Breast cancer is considered a public health problem with an increasing incidence worldwide. Several factors contribute to late diagnosis and hinder the initiation of the treatment, resulting in a worse prognosis. Objectives: To analyze the time interval between diagnosis and the beginning of the first oncologic treatment in the Brazilian population, in addition to assessing the factors associated with the longest time intervals. Method: Retrospective cohort study with 540,529 patients registered in the Hospital Cancer Registry System (SisRHC) from 2000 to 2017. The outcome was the time between diagnosis and the beginning of the first oncologic treatment, considering the interval greater than 60 days as delay. Descriptive analysis and simple logistic regression were performed ( $95 \%$ CI; p <0.05) to analyze the variables. Results: 204,130 cases were analyzed, mean age of 55.8 years $( \pm 13.24)$, predominantly females $(99.1 \%), 55.1 \%$ were from the southeast region and $71 \%$ lived in non-capital cities. The median of the time interval between diagnosis and the beginning of the first oncologic treatment was 63 days (interquartile range $=36-109$ ). Sociodemographic, clinical and treatment-related variables affect the time interval, except the gender variable. Conclusion: The time between diagnosis and the beginning of the first oncologic treatment was high. Sociodemographic, clinical and treatment-related factors influence time intervals. Their early identification can contribute to guide the actions toward these most vulnerable groups to delay.

Key words: Breast Neoplasms/diagnosis; Time-to-Treatment; Risk Factors; Hospital Records.
RESUMEN

Introducción: El cáncer de mama se considera un problema de salud pública con una incidencia mundial creciente. Varios factores contribuyen al diagnóstico tardío y dificultan el inicio del tratamiento, resultando en un peor pronóstico. Objetivos: Analizar el intervalo de tiempo entre el diagnóstico y el comienzo del primer tratamiento oncologico en la población brasileńa, además de evaluar los factores asociados con los intervalos de tiempo más largos. Método: Este es un estudio de cohorte retrospectivo con 540.529 pacientes registrados en el Hospital Cancer Registry System (SisRHC) desde 2000 hasta 2017. El resultado fue el intervalo de tiempo entre el diagnóstico yel comienzo del primer tratamiento oncologico, considerando como retraso el intervalo superior a 60 días. Para el análisis de las variables, se realizó un análisis descriptivo y una regresión logística simple (IC95\%; $\mathrm{p}<0,05)$. Resultados: Se analizaron 204,130 casos, con una edad media de 55,8 ańos $( \pm 13,24)$, predominantemente mujeres $(99,1 \%)$, $55,1 \%$ dela región surestey $71,4 \%$ residentes en ciudades no capitales. La mediana del intervalo de tiempo entre el diagnóstico y el comienzo del primer tratamiento contra el cáncer fue de 63 días (rango intercuartil = 36-109). Las variables sociodemográficas, clínicas y relacionadas con el tratamiento tuvieron un impacto en el intervalo de tiempo, con la excepción dela variable de género. Conclusión: El intervalo de tiempo promedio entre el diagnóstico y el comienzo del primer tratamiento oncologico fue alto. Además, se observó que los factores sociodemográficos, clínicos y relacionados con el tratamiento influyen en los intervalos de tiempo, por lo que identificarlos temprano puede contribuir a acciones directas para estos grupos más vulnerables al retraso.

Palabras clave: Neoplasias dela Mama/diagnóstico; Tiempo de Tratamiento; Factores de Riesgo; Registros de Hospitales.

${ }^{1}$ Coordenação de Pesquisa Clínica do Instituto Nacional de Câncer José Alencar Gomes da Silva (INCA). Rio de Janeiro (RJ), Brasil. Orcid iD: https://orcid.org/0000-0002-4924-866X ${ }^{2}$ Coordenação de Pesquisa Clínica do INCA. Rio de Janeiro (RJ), Brasil. Orcid iD: https://orcid.org/0000-0002-7029-2198

${ }^{3}$ Coordenação de Pesquisa Clínica do INCA. Rio de Janeiro (RJ), Brasil. Orcid iD: https://orcid.org/0000-0003-0959-7678

${ }^{4}$ Coordenação de Pesquisa Clínica do INCA. Rio de Janeiro (RJ), Brasil. Orcid iD: https://orcid.org/0000-0003-1963-1294

${ }^{5}$ Universidade Federal de Campina Grande (UFCG). Campina Grande (PB), Brasil. Orcid iD: https://orcid.org/0000-0003-2923-9595

${ }^{6}$ Universidade Federal do Rio de Janeiro (UFRJ). Campus Macaé. Macaé (RJ), Brasil. Orcid iD: https://orcid.org/0000-0002-9022-1848

${ }^{7}$ Universidade Federal de Santa Maria (UFSM). Santa Maria (RS), Brasil. Orcid iD: https://orcid.org/0000-0001-5103-7135

${ }^{8}$ Universidade Federal do Maranhão (UFMA). São Luís (MA), Brasil. Orcid iD: https://orcid.org/0000-0001-9654-7999

${ }^{9}$ Universidade Estadual do Paraná (Unespar). Campus de Paranavaí. Paranavaí (PR), Brasil. Orcid iD: https://orcid.org/0000-0002-7170-3315

${ }_{10}$ Universidade Federal do Espírito Santo (UFES). Campus Maruípe. Vitória (ES), Brasil. Orcid iD: https://orcid.org/0000-0002-8372-325X

${ }^{11}$ Coordenação de Pesquisa Clínica do INCA. Rio de Janeiro (RJ), Brasil. Orcid iD: https://orcid.org/0000-0002-1972-8777

${ }^{12}$ Coordenação de Pesquisa Clínica do INCA. Rio de Janeiro (RJ), Brasil. Orcid iD: https://orcid.org/0000-0003-2550-6537

Endereço para correspondência: Luiz Claudio Santos Thuler. Rua André Cavalcanti, 37 - sala 9 (anexo) - Centro. Rio de Janeiro (RJ), Brasil. CEP 20231-050. E-mail:Ithuler@gmail.com 


\section{INTRODUÇÃO}

O câncer de mama (CM) é considerado um problema de saúde pública ${ }^{1}$. Ocupa a segunda posição entre os tipos de cânceres com maior incidência mundial, estando em primeiro lugar entre o sexo feminino, excetuando-se os cânceres de pele não melanoma². Em 2018, foram estimados 2,1 milhóes de novos casos de CM no mundo e mais de 626 mil óbitos pela doença. As maiores taxas de incidência de CM são observadas em países desenvolvidos (41,1 por $100 \mathrm{mil}$ ) quando comparados aos países em desenvolvimento (32,8 por 100 mil), embora haja uma inversão desse panorama com relação às taxas de mortalidade, que são de 17,1 e 10,3 por 100 mil, respectivamente ${ }^{2}$.

No Brasil, estimam-se 66.280 casos novos de CM para cada ano do triênio 2020-2022, sendo o tipo de câncer mais incidente entre as mulheres nas Regiốes Sul, Sudeste, Centro-Oeste e Nordeste, sem considerar os tumores de pele não melanoma ${ }^{1}$. $\mathrm{O} \mathrm{CM}$ é a principal causa de morte por câncer entre as mulheres em quatro das cinco Macrorregiôes do Brasil, com exceção da Regiáo Norte, onde ocupa o segundo lugar ${ }^{3}$. A taxa de sobrevida em cinco anos, no país, é de aproximadamente $68,7 \%{ }^{4}$. Entre 1988 e 2008, a taxa de incidência de CM entre a população masculina brasileira triplicou ${ }^{5}$. Características sociodemográficas, clínicas e relacionadas ao tratamento diferem significativamente entre homens e mulheres diagnosticados com CM no país, apesar do prognóstico semelhante ${ }^{6}$.

As disparidades entre países desenvolvidos e em desenvolvimento são atribuídas a vários fatores clínicos e sociodemográficos, que resultam na falta de acesso da população aos serviços de saúde ${ }^{7-9}$. Esse contexto contribui para o diagnóstico tardio do $\mathrm{CM}^{4,5}$. Além disso, barreiras sociais, geográficas e relacionadas ao serviço de saúde favorecem o atraso para o início do tratamento ${ }^{7,10}$. Apesar das incertezas sobre o verdadeiro impacto do atraso na sobrevida de mulheres com CM, estudos têm mostrado que os longos intervalos de tempo entre o diagnóstico de CM e o início do primeiro tratamento estão associados a pior prognóstico ${ }^{11,12}$.

No Brasil, em 2013, foi publicada a Portaria ${ }^{\circ}$. $876 / 13^{13}$, que dispóe sobre a aplicaçáo da Lei no. $12.732 / 12$, que estabelece o prazo de até 60 dias para o início do tratamento oncológico após confirmação diagnóstica no âmbito do Sistema Único de Saúde (SUS) ${ }^{14}$.

A elevada proporçáo de mulheres com estadiamento clínico (EC) avançado ao diagnóstico e o aumento crescente nas taxas de mortalidade indicam que as açóes estabelecidas para o controle do CM no Brasil podem não estar sendo eficazes ${ }^{15,16}$. Pesquisadores brasileiros observaram que, na maioria dos casos, o primeiro tratamento oncológico é iniciado com atraso ${ }^{17-19}$. Os entraves no acesso à assistência oncológica no país têm consequências significativas para a saúde dessa populaçáo $2^{20,21}$.

Nesse contexto, o objetivo do presente estudo é analisar o intervalo de tempo entre o diagnóstico e o início do primeiro tratamento oncológico na população brasileira, além de avaliar os fatores associados ao seu atraso.

\section{MÉTODO}

Trata-se de um estudo de coorte retrospectivo com dados secundários oriundos do Sistema de Registros Hospitalares de Câncer (SisRHC) do Brasil. As informaçôes foram obtidas por meio de download da base de dados provenientes do sistema web Integrador RHC, realizado em 30 de novembro de 2019. Foram incluídos pacientes com CM, diagnosticados e tratados no período de 2000 a 2017. Os casos registrados nesse banco de dados são provenientes de 336 unidades hospitalares. Os critérios de exclusão foram: casos não analíticos (casos diagnosticados e tratados - parcial ou totalmente - fora do hospital onde está instalado o RHC e casos matriculados no hospital do RHC, mas que náo iniciam tratamento antineoplásico na unidade $)^{22}$; idade $<18$ ou $>99$ anos; casos sem informaçáo sobre o sexo; pacientes com câncer não invasivo; sem informação de EC ou com EC IV, cujo primeiro tratamento foi instituído com finalidade paliativa; sem informação da data do diagnóstico ou tratamento; aqueles que não realizaram tratamento ou que não possuíam informação sobre o primeiro tratamento realizado; casos em que o tratamento foi datado como anterior ao diagnóstico (tempo negativo) e com intervalo de tempo superior a 365 dias.

As variáveis sociodemográficas utilizadas para análise foram: sexo; faixa etária; raça/cor de pele, de acordo com o Instituto Brasileiro de Geografia e Estatística (IBGE); escolaridade; Índice de Desenvolvimento Humano Municipal (IDHM), identificado a partir do código de município e classificado de acordo com o Programa das Naçôes Unidas para o Desenvolvimento (PNUD) ${ }^{23}$; estado conjugal; consumo de álcool; consumo de tabaco; cidade de procedência; regiáo de residência; região da unidade hospitalar; e origem de encaminhamento (SUS e outros). As variáveis clínicas e relacionadas ao primeiro tratamento foram: o tipo histológico; EC; ano de diagnóstico (categorizados nos períodos pré-lei: 2000-2005 e 2006-2011 e pós-lei: 2012-2017); cidade de residência e tratamento; unidade de tratamento; e o primeiro tratamento oncológico realizado. 
Foi estabelecido como desfecho do estudo o intervalo de tempo entre o diagnóstico e o início do primeiro tratamento oncológico, com intenção curativa. O intervalo de tempo superior a 60 dias foi definido como atraso, baseando-se na Portaria $n^{\circ} .876 / 13^{13}$.

Foi realizada análise descritiva da populaçáo do estudo. As variáveis contínuas foram analisadas por meio de distribuição (frequências absoluta e relativa) e medidas de tendência central (média e mediana). A análise da associação entre os fatores sociodemográficos e clínicos com o desfecho (atraso) foi realizada utilizando a regressão logística simples. Foram consideradas estatisticamente significantes as variáveis com $\mathrm{p}<0,05$. Os resultados foram descritos utilizando odds ratio (OR) e considerando intervalo de confiança de 95\% (IC 95\%). Os dados foram analisados por meio do pacote estatístico Statistical Package for the Social Sciences (SPSS) versão 23.0 (IBM Corp., Armonk, Estados Unidos).

\section{RESULTADOS}

Inicialmente, foram incluídas 540.529 pessoas diagnosticadas com CM, registradas no SisRHC, que iniciaram tratamento oncológico nos anos de 2000 a 2017. Após aplicação dos critérios de elegibilidade, foram excluídos 322.891 casos, totalizando uma população final de 204.130 para análise (Figura 1). Os pacientes apresentaram média de idade de 55,8 $( \pm 13,24)$ anos, com predomínio do sexo feminino (99,1\%); 55,1\% eram da Regiáo Sudeste; 49,6\% apresentavam menos de oito anos de estudo; 44,0\% tinham como origem de encaminhamento o SUS; e $71,4 \%$ residiam em cidade fora da capital (Tabela 1).

A população foi composta, em sua maioria $(87,4 \%)$, por pacientes com carcinoma ductal invasivo (CDI), 58,8\% realizaram cirurgia como primeiro tratamento, $52,0 \%$ apresentavam estadiamento avançado ao diagnóstico, $56,5 \%$ foram tratados em "outras unidades" e 54,8\% foram tratados fora da cidade de residência (Tabela 2). Nesse banco de dados, foram identificados 43 Centros de Assistência de Alta Complexidade em Oncologia (Cacon) $(14,5 \%)$ e 252 "outras unidades" (85,4\%) que realizaram tratamento para o CM (dados não tabulados).

$\mathrm{O}$ intervalo de tempo mediano entre o diagnóstico e o primeiro tratamento foi de 63 dias (variação interquartil [VIQ]: 36-109), sendo que 52,8\% dos pacientes apresentaram o intervalo de tempo superior a 60 dias.

Pode-se observar que todas as variáveis sociodemográficas e clínicas apresentaram associação estatisticamente significante com o atraso, com exceçáo da variável sexo ( $\mathrm{OR}=0,99 ; \mathrm{IC}=0,91-1,09 ; \mathrm{p}=0,862)$. Os pacientes submetidos à radioterapia e à hormonioterapia como primeiro tratamento apresentaram 2,11 (IC95\% 2,04$2,18 ; \mathrm{p}<0,001)$ e 2,13 (IC95\% 2,03-2,24; $<<0,001$ ) maior chance de atraso para o início do tratamento, respectivamente, quando comparados aos submetidos à cirurgia. Os pacientes diagnosticados no período de 2006-2011 e 2012-2017 apresentaram maior chance de atraso se comparados àqueles diagnosticados entre 2000-2005 (Tabela 2).

A Figura 2 ilustra a distribuição do número de casos registrados no RHC por ano. Observa-se que houve um aumento gradativo ao longo do período de 17 anos, com um aumento expressivo em 2013 e 2014.

Na Figura 3, é possível observar que, entre os anos de 2000 e 2011, a frequência de casos que apresentaram intervalo entre o diagnóstico e o tratamento superior a 60 dias aumentou gradativamente ao longo do tempo. Em 2012, observou-se o pico do atraso, indicando que quase $60 \%$ dos indivíduos tiveram acesso à terapia oncológica mais de 60 dias após terem recebido o diagnóstico de CM. Esse atraso permaneceu nos anos subsequentes; no entanto, desde 2015, parece estar havendo discreta redução na demora, embora os percentuais se mantenham elevados.

\section{DISCUSSÃO}

No presente estudo, constatou-se que o atraso foi mais frequente $(46,4 \%)$ entre as pacientes diagnosticadas de 2012 a 2017, sendo esse o período em que a Lei no. $12.732 / 12^{14}$, também conhecida como "Lei dos 60 dias", passou a vigorar. $\mathrm{Na}$ análise de regressão logística simples, foi verificado que ter recebido o diagnóstico de CM após a publicação da Lei aumentou em 64\% a chance de atraso para o início do tratamento quando comparado ao período de 2000 a 2005. Medeiros et al. ${ }^{9}$ analisaram dados nacionais oriundos do RHC e sugeriram que o aumento do atraso entre 2006 a 2011, em relação ao período de 2000 a 2005, pode estar relacionado ao aumento da incidência de CM no país, juntamente com a inadequaçáo no aumento da oferta de serviços especializados necessários para suprir a demanda por atendimento9. Em 2011, em um levantamento realizado pela Divisão de Detecção Precoce e Apoio à Organização de Rede do Instituto Nacional de Câncer José Alencar Gomes da Silva (INCA), foi verificado que a capacidade instalada e a produçấo da rede oncológica necessária para todo o território nacional era de 375 Cacon ou Unidades de Assistência de Alta Complexidade em Oncologia (Unacon). Entretanto, a capacidade real era cerca de 30\% menor do que o necessário, com 264 estabelecimentos habilitados à época. O déficit da capacidade instalada juntamente com um pico no número de casos registrados 
Tabela 1. Variáveis sociodemográficas de pacientes com câncer de mama. Registros Hospitalares de Câncer. Brasil, $2000-2017$ (N=204.130)

\begin{tabular}{|c|c|c|c|c|c|}
\hline \multirow{2}{*}{ Variáveis } & \multirow{2}{*}{$\begin{array}{c}\text { Total } \\
204.130 \\
(100,0)\end{array}$} & \multicolumn{2}{|c|}{$\begin{array}{l}\text { Tempo } \\
\mathbf{N}(\%)\end{array}$} & \multirow{2}{*}{ OR (IC95\%) } & \multirow{2}{*}{$\begin{array}{l}\text { Valor } \\
\text { de } p\end{array}$} \\
\hline & & $\begin{array}{c}\geq 61 \text { dias } \\
107.741(52,8)\end{array}$ & $\begin{array}{c}0 \text { a } 60 \text { dias } \\
96.389(47,2)\end{array}$ & & \\
\hline \multicolumn{6}{|l|}{ Sexo } \\
\hline Masculino & $1.931(0,9)$ & $1.023(0,9)$ & $908(0,9)$ & Referência & \\
\hline Feminino & $202.199(99,1)$ & $106.718(99,1)$ & $95.481(99,1)$ & $0,99(0,91-1,08)$ & 0,862 \\
\hline \multicolumn{6}{|l|}{ Idade } \\
\hline$\leq 49$ anos & $75.771(37,1)$ & $37.126(34,5)$ & $38.645(40,1)$ & Referência & \\
\hline 50 a 69 anos & $96.935(47,5)$ & $52.750(49,0)$ & $44.185(45,8)$ & $1,24(1,22-1,27)$ & $<0,001$ \\
\hline 70 anos ou mais & $31.424(15,4)$ & $17.865(16,6)$ & $13.559(14,1)$ & $1,37(1,34-1,41)$ & $<0,001$ \\
\hline \multicolumn{6}{|l|}{ Raça/cor da pele } \\
\hline Branca & $65.556(32,1)$ & $34.290(31,8)$ & $31.266(32,4)$ & Referência & \\
\hline Não branca* & $58.543(28,7)$ & $31.337(29,1)$ & $27.206(28,2)$ & $1,05(1,03-1,07)$ & 0,001 \\
\hline Sem informação & $80.031(39,2)$ & $42.114(39,1)$ & $37.917(39,3)$ & $1,01(0,99-1,03)$ & 0,230 \\
\hline \multicolumn{6}{|l|}{ Estado conjugal } \\
\hline Com companheiro & $64.185(31,4)$ & $33.327(30,9)$ & $30.858(32,0)$ & Referência & \\
\hline Sem companheiro & $56.145(27,5)$ & $30.580(28,4)$ & $25.565(26,5)$ & $1,11(1,08-1,13)$ & $<0,001$ \\
\hline Sem informação & $83.800(41,1)$ & $43.834(40,7)$ & $39.966(41,5)$ & $1,02(0,99-1,04)$ & 0,142 \\
\hline \multicolumn{6}{|l|}{ Consumo de álcool } \\
\hline Nunca & $72.781(35,7)$ & $38.520(35,8)$ & $34.261(35,5)$ & Referência & \\
\hline Ex ou consumo atual & $16.031(7,9)$ & $9.053(8,4)$ & $6.978(7,2)$ & $1,15(1,11-1,19)$ & $<0,001$ \\
\hline Sem informação & $115.318(56,5)$ & $60.168(55,8)$ & $55.150(57,2)$ & $0,97(0,95-0,99)$ & 0,002 \\
\hline \multicolumn{6}{|l|}{ Consumo de tabaco } \\
\hline Nunca & $68.063(33,3)$ & $35.982(33,4)$ & $32.081(33,3)$ & Referência & \\
\hline Ex ou consumo atual & $28.496(14,0)$ & $15.542(14,4)$ & $12.954(13,4)$ & $1,07(1,04-1,10)$ & $<0,001$ \\
\hline Sem informação & $107.571(52,7)$ & $56.217(52,2)$ & $51.354(53,3)$ & $0,98(0,96-0,99)$ & 0,013 \\
\hline \multicolumn{6}{|l|}{ Região de residência } \\
\hline Sul & $36.128(17,7)$ & $18.671(17,4)$ & $17.457(18,2)$ & Referência & \\
\hline Sudeste & $112.381(55,2)$ & $60.609(56,4)$ & $51.772(53,9)$ & $1,09(1,07-1,12)$ & $<0,001$ \\
\hline Centro-Oeste & $5.355(2,6)$ & $2.882(2,7)$ & $2.473(2,6)$ & $1,09(1,03-1,15)$ & 0,003 \\
\hline Nordeste & $42.917(21,1)$ & $21.166(19,7)$ & $21.751(22,6)$ & $0,91(0,88-0,94)$ & $<0,001$ \\
\hline Norte & $6.821(3,4)$ & $4.148(3,9)$ & $2.673(2,8)$ & $1,45(1,38-1,53)$ & $<0,001$ \\
\hline \multicolumn{6}{|l|}{ Origem encaminhamento } \\
\hline SUS & $89.784(44,0)$ & $49.298(45,8)$ & $40.486(42,0)$ & Referência & \\
\hline Outros meios & $29.874(14,6)$ & $14.639(13,6)$ & $15.235(15,8)$ & $0,79(0,77-0,81)$ & $<0,001$ \\
\hline Sem informação & $84.472(41,4)$ & $43.804(40,7)$ & $40.668(42,2)$ & $0,88(0,87-0,90)$ & $<0,001$ \\
\hline \multicolumn{6}{|l|}{ Cidade procedência** } \\
\hline Capital & $58.269(28,6)$ & $31.521(29,3)$ & $26.748(27,8)$ & Referência & \\
\hline Não capital & $145.333(71,4)$ & $75.955(70,7)$ & $69.378(72,2)$ & $0,93(0,91-0,95)$ & $<0,001$ \\
\hline \multicolumn{6}{|l|}{$\begin{array}{l}\text { IDHM da cidade de } \\
\text { procedência*** }\end{array}$} \\
\hline Muito alto $(0,800-1,000)$ & $43.337(21,3)$ & $21.754(20,2)$ & $21.583(22,5)$ & Referência & $<0,001$ \\
\hline Outros $^{£}(0,000-0,779)$ & $160.249(78,7)$ & $85.713(79,8)$ & $74.536(77,5)$ & $1,14(1,12-1,16)$ & \\
\hline
\end{tabular}

Legendas: *Não branca: preta, parda, amarela e indígena; ${ }^{* *}$ Capital: corresponde às 26 capitais brasileiras e Distrito Federal; Não capital: demais cidades; ${ }^{* * *} \mathrm{IDHM}=$ Índice de Desenvolvimento Humano Municipal; ${ }^{£}=$ Outros: Muito baixo, baixo, médio, alto; OR = Odds ratio; IC = Intervalo de confiança.

no RHC em 2013 e 2014 podem justificar os maiores atrasos nesse período ${ }^{24}$. Cabe ressaltar que a Portaria ${ }^{\circ}$. $140 / 14^{25}$, que estabelece a obrigatoriedade de atualização das informaçôes referentes ao RHC, por parte dos estabelecimentos de saúde habilitados como Cacon ou
Unacon, pode ter aumentado o número de registros e melhorado a qualidade dos dados ${ }^{25}$. Em 2011, na auditoria realizada pelo INCA, foi observado que menos da metade dos estabelecimentos enviaram dados sobre o tempo de espera para realização de procedimentos ${ }^{24}$. 
Tabela 2. Variáveis clínicas e relacionadas ao tratamento de pacientes com câncer de mama. Registros Hospitalares de Câncer. Brasil, 2000$2017(\mathrm{~N}=204.130)$

\begin{tabular}{|c|c|c|c|c|c|}
\hline \multirow{2}{*}{ Variáveis } & \multirow{2}{*}{$\begin{array}{c}\text { Total } \\
204.130 \\
(100,0)\end{array}$} & \multicolumn{2}{|c|}{$\begin{array}{l}\text { Tempo } \\
\text { N (\%) }\end{array}$} & \multirow{2}{*}{ OR (IC95\%) } & \multirow{2}{*}{$\begin{array}{l}\text { Valor } \\
\text { de } p\end{array}$} \\
\hline & & $\begin{array}{c}\leq 61 \text { dias } \\
107.741(52,8)\end{array}$ & $\begin{array}{c}0 \text { a } 60 \text { dias } \\
96.389(47,2)\end{array}$ & & \\
\hline \multicolumn{6}{|l|}{ Tipo histológico } \\
\hline CDI & $178.384(87,4)$ & $93.728(87,0)$ & $84.656(87,8)$ & Referência & \\
\hline Outros & $25.746(12,6)$ & $14.013(13,0)$ & $11.733(12,2)$ & $1,08(1,05-1,10)$ & $<0,001$ \\
\hline \multicolumn{6}{|l|}{ Primeiro tratamento } \\
\hline Cirurgia & $119.929(58,8)$ & $62.992(58,5)$ & $56.937(59,1)$ & Referência & \\
\hline Radioterapia & $17.921(8,8)$ & $12.544(11,6)$ & $5.377(5,6)$ & $2,11(2,04-2,18)$ & $<0,001$ \\
\hline Quimioterapia & $57.624(28,2)$ & $26.255(24,4)$ & $31.369(32,5)$ & $0,75(0,74-0,77)$ & $<0,001$ \\
\hline Hormonioterapia & $8.115(4,0)$ & $5.699(5,3)$ & $2.416(2,5)$ & $2,13(2,03-2,24)$ & $<0,001$ \\
\hline Outras & $541(0,3)$ & $251(0,2)$ & $290(0,3)$ & $0,78(0,66-0,93)$ & 0,004 \\
\hline \multicolumn{6}{|l|}{ Estadiamento clínico } \\
\hline Inicial (0 a IIA) & $97.961(48,0)$ & $55.847(51,8)$ & $42.114(43,7)$ & Referência & \\
\hline Avançado (IIB a IIIC) & $106.169(52,0)$ & $51.894(48,2)$ & $54.275(56,3)$ & $0,72(0,71-0,93)$ & $<0,001$ \\
\hline \multicolumn{6}{|c|}{ Unidade de tratamento } \\
\hline Outras unidades* & $115.359(56,5)$ & $58.530(54,3)$ & $56.829(59,0)$ & Referência & \\
\hline Cacon & $88.771(43,5)$ & $49.211(45,7)$ & $39.560(41,0)$ & $1,20(1,18-1,22)$ & $<0,001$ \\
\hline \multicolumn{6}{|c|}{$\begin{array}{l}\text { Mesma cidade de } \\
\text { residência e tratamento }\end{array}$} \\
\hline Sim & $91.778(45,1)$ & $46.555(43,3)$ & $45.223(47,0)$ & Referência & $<0,001$ \\
\hline Não & $111.824(54,9)$ & $60.921(56,7)$ & $50.903(53,0)$ & $1,16(1,14-1,18)$ & \\
\hline \multicolumn{6}{|l|}{ Ano do diagnóstico } \\
\hline 2000-2005 & $41.671(20,4)$ & $18.218(16,9)$ & $23.453(24,3)$ & Referência & \\
\hline 2006-2011 & $73.320(35,9)$ & $39.532(36,7)$ & $33.788(35,1)$ & $1,51(1,47-1,54)$ & $<0,001$ \\
\hline 2012-2017 & $89.139(43,7)$ & $49.991(46,4)$ & $39.148(40,6)$ & $1,64(1,61-1,68)$ & $<0,001$ \\
\hline \multicolumn{6}{|l|}{$\begin{array}{l}\text { Região da unidade } \\
\text { hospitalar }\end{array}$} \\
\hline Sul & $36.344(17,8)$ & $18.778(17,4)$ & $17.566(18,2)$ & Referência & \\
\hline Sudeste & $114.665(56,2)$ & $62.082(57,6)$ & $52.583(54,6)$ & $1,10(1,08-1,13)$ & $<0,001$ \\
\hline Centro-Oeste & $4.287(2,1)$ & $2.076(1,9)$ & $2.211(2,3)$ & $0,88(0,82-0,94)$ & $<0,001$ \\
\hline Nordeste & $42.609(20,9)$ & $20.996(19,5)$ & $21.613(22,4)$ & $0,91(0,88-0,94)$ & $<0,001$ \\
\hline Norte & $6.225(3,0)$ & $3.809(3,5)$ & $2.416(2,5)$ & $1,47(1,40-1,56)$ & $<0,001$ \\
\hline
\end{tabular}

Legendas: $\mathrm{CDI}=$ Carcinoma ductal invasivo; ${ }^{*}$ Outras unidades: Unacon = Unidades de Alta Complexidade em Oncologia, serviços de radioterapia e hospitais gerais; Cacon = Centros de Alta Complexidade em Oncologia; OR = Odds ratio; IC = Intervalo de confiança.

No Brasil, o aumento nas açôes de rastreamento do CM náo estão impactando positivamente na redução da mortalidade. Isso porque, além da baixa cobertura mamográfica entre a população-alvo, existe uma inadequação no seguimento de lesôes suspeitas e déficit de acesso a tratamento cirúrgico ${ }^{20}$.

Vale destacar que, apesar da "Lei dos 60 dias" representar um progresso na atenção oncológica no Brasil, os resultados do atual estudo indicam que a legislação não está sendo cumprida. Sousa et al. ${ }^{17}$ observaram que $71,6 \%$ das mulheres com CM diagnosticadas e tratadas no Estado do Piauí, entre 2016 e 2017, apresentaram atraso superior a 60 dias. Esses autores sugerem que a desarticulaçáo entre os serviços de saúde impede que pacientes com CM tenham acesso ao seu direito garantido por Lei. Em contrapartida, em um estudo realizado em Belo Horizonte ${ }^{18}$, a partir de uma coorte de pacientes cadastradas no RHC, diagnosticadas no período de 2010 a 2013, foi verificado que pouco mais da metade da população estudada $(54,3 \%)$ apresentou intervalo de até 60 dias entre o diagnóstico e o início de tratamento; no entanto, foi constatado que, quanto maior o perfil de vulnerabilidade social das mulheres, maior a chance de atraso $(\mathrm{p}<0,001)$. Esse resultado revela a existência 


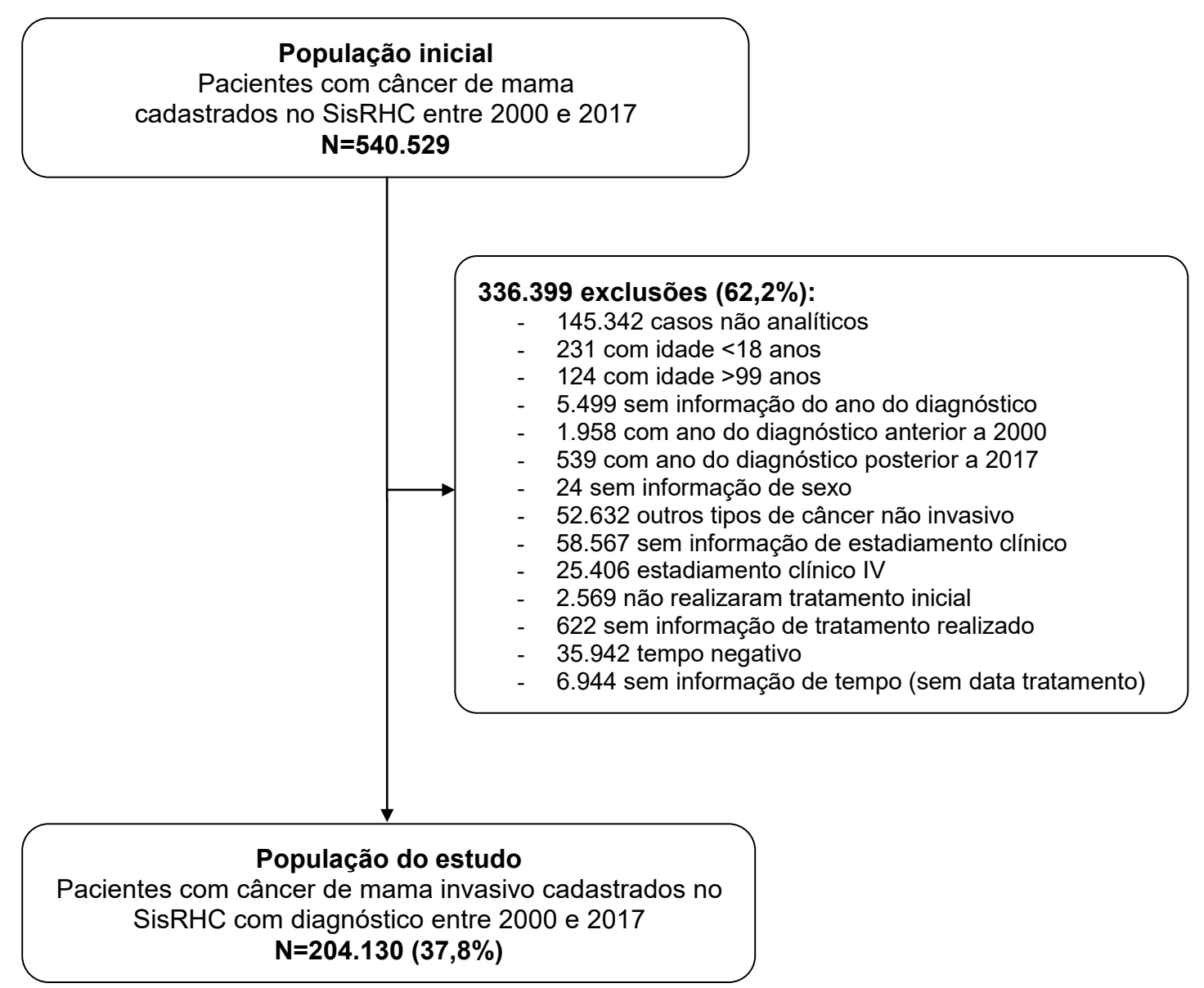

Figura 1. Diagrama de fluxo com os critérios de inclusão e exclusão do estudo

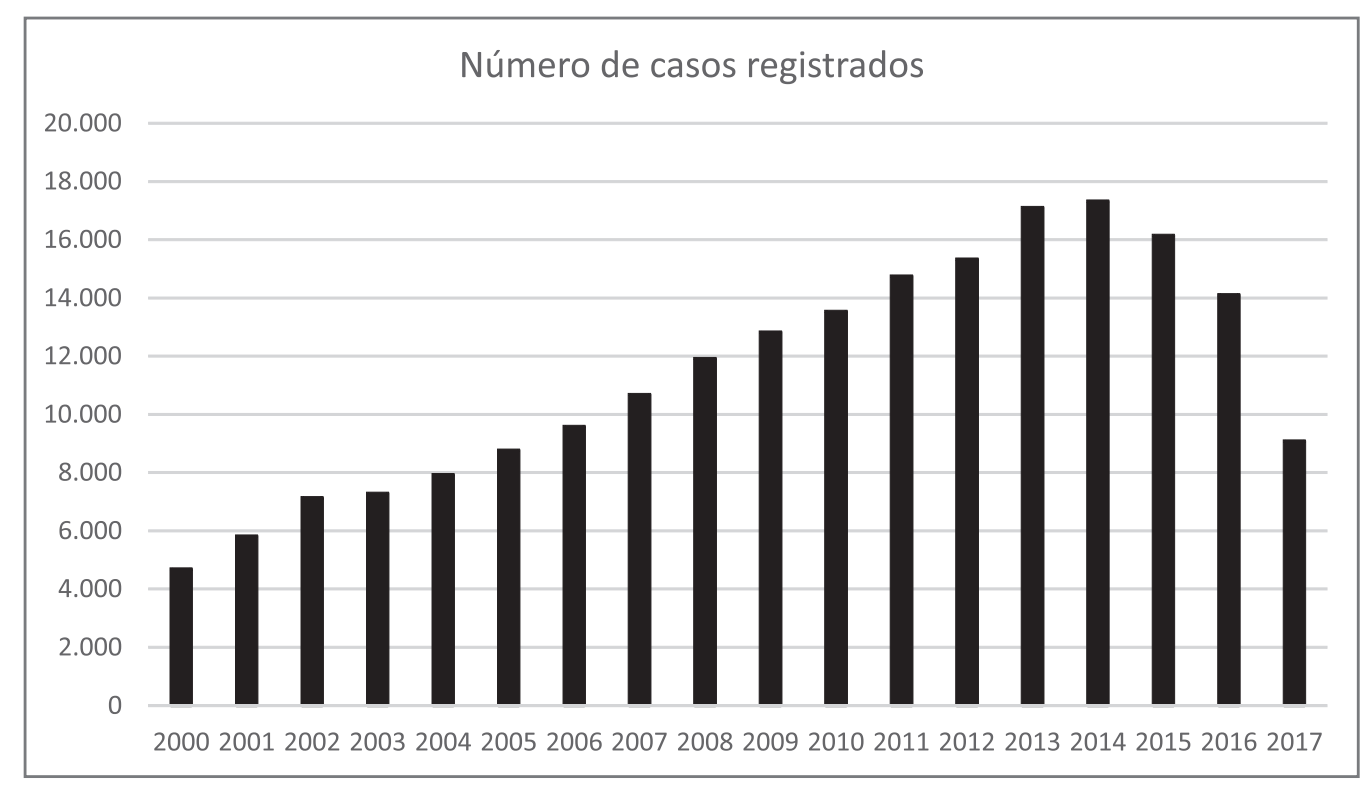

Figura 2. Distribuição absoluta do número de casos registrados nos Registros Hospitalares de Câncer por ano do diagnóstico, no período de 2000-2017 ( $N=204.130)$ 


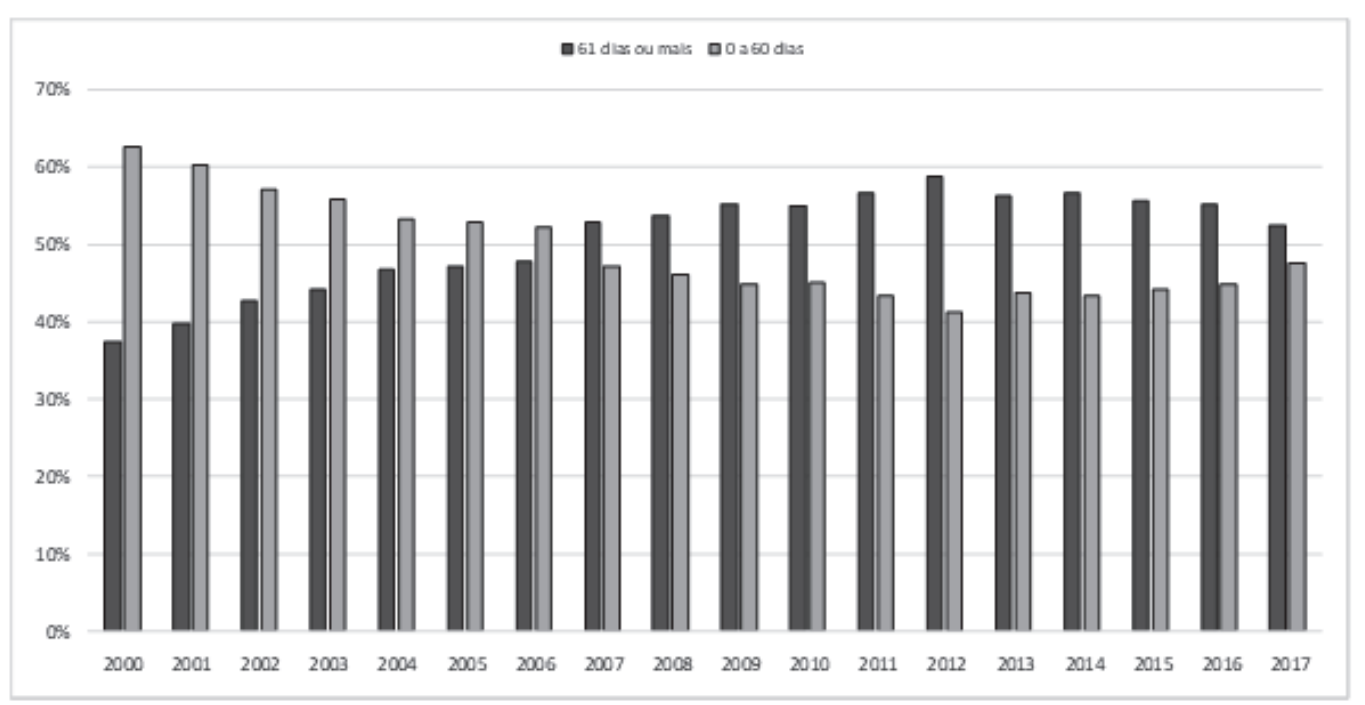

Figura 3. Distribuição de frequência dos intervalos de tempo entre o diagnóstico e o início do tratamento, Registros Hospitalares de Câncer. Brasil, 2000-2017 ( $\mathrm{N}=204.130)$

de inequidades no acesso ao tratamento do CM no país, mesmo após barreiras no acesso às unidades de saúde terem sido, aparentemente, ultrapassadas ${ }^{18}$.

Em outros países, os intervalos de tempo observados foram inferiores ao identificado no presente estudo ${ }^{26-28}$. McLaughlin et al. ${ }^{26}$ observaram que, na população de mulheres na Carolina do Norte (EUA), a mediana de tempo entre o diagnóstico e o tratamento foi de 22 dias (mínimo: 0, máximo: 177 dias). A maioria das pacientes $(90 \%)$ iniciou o tratamento em um intervalo de tempo menor que 60 dias e, em $81 \%$ dos casos, a cirurgia foi o primeiro tratamento realizado. Comparando os resultados, observa-se que há uma grande discrepância entre os estudos, que pode estar relacionada às diferenças nas estruturas dos serviços de saúde entre os países. No entanto, apesar dessa responsabilidade ser, frequentemente, atribuída aos entraves existentes no âmbito institucional, Travassos e $\mathrm{Bahia}^{29}$ sugerem que essa realidade é derivada da relação profissional saúde-paciente.

Apesar de não ter sido identificada associação entre sexo e atraso, possivelmente, essa diferença entre homens e mulheres podem ser visualizadas nas etapas anteriores ao diagnóstico ${ }^{30}$. Em virtude da raridade da doença na população masculina, pode-se esperar que haja demora na suspeição da doença, por parte do paciente e do profissional de saúde, retardando a busca pela assistência e o início da investigação diagnóstica ${ }^{31}$. Além disso, métodos e linhas de tratamento de $\mathrm{CM}$ para os homens ainda são baseados nos realizados para a população feminina, o que justifica as semelhanças entre os sexos ${ }^{32}$. Em um estudo recente, foi constatado que a média anual da incidência de $\mathrm{CM}$ em homens aumentou consideravelmente ${ }^{33}$. Diante desse fato, destaca-se a importância de analisar o fluxo de atendimento a homens diagnosticados com $\mathrm{CM}$, desde o início dos sintomas até o acesso ao serviço de saúde, a fim de tornar esses processos mais eficazes para pacientes desse sexo.

Pacientes da raça/cor da pele não branca, com menos de oito anos de estudo, com idade entre 50 e 69 anos e que viviam sem companheiro(a) apresentaram maior chance de atraso quando comparados aos de outras categorias. Esses fatores já têm sido comumente associados ao atraso ${ }^{7,9,10}$. Os resultados observados podem ser atribuídos às desigualdades socioeconômicas e étnicas, que configuram um perfil de maior vulnerabilidade social, impactando negativamente na busca e no acesso aos serviços de saúde ${ }^{18,34}$. A estrutura familiar e a rede de apoio social também influenciam nesse contexto ${ }^{35,36}$.

$\mathrm{O}$ fato de pacientes com $\mathrm{EC} \geq 2 \mathrm{~B}$ terem apresentado menor chance de atraso do que aqueles com doença inicial, assim como observado por outros autores, reforça a hipótese de que os médicos agem mais rapidamente diante dos casos de maior gravidade ${ }^{11}$. No presente estudo, o EC avançado ( $\geq 2 \mathrm{~B}$ ) foi o mais frequente $(52,0 \%)$. Esse dado é corroborado por outros autores que indicam que, no Brasil, a maioria dos casos é diagnosticado com doença avançada ${ }^{9}$. Essa realidade é alarmante, pois sabe-se que o EC constitui um importante fator prognóstico. Como visto por Höfelmann et al. ${ }^{37}$, em um estudo realizado em Santa Catarina, houve uma diminuição da sobrevida com o aumento do estadiamento (EC I: 98\%; EC II: $81 \%$; EC III e EC IV: 60\%). Além disso, essa característica clínica interfere na qualidade de vida de indivíduos com CM. Isto é, pacientes diagnosticados em estádios iniciais são submetidos a tratamentos menos agressivos, e apresentam menos complicaçôes relacionadas à terapia oncológica e, 
consequentemente, menor risco de morte ${ }^{38}$. Diante dessa problemática, ressalta-se a importância de priorizar as políticas de rastreamento, diagnóstico precoce e garantia do tratamento em tempo hábil, com vistas ao aumento da sobrevida e melhores desfechos ${ }^{15,20}$.

As categorias de tratamento, baseadas nas condutas terapêuticas que ocorreram de acordo com EC, diferem com relação à probabilidade de exposição ao atraso. Quando comparados aos submetidos à cirurgia tratamento mais frequente neste estudo - observou-se que pacientes que realizaram quimioterapia e radioterapia tiveram, aproximadamente, duas vezes maior chance de atraso. No Brasil, existem desigualdades na oferta de assistência especializada para fins de diagnóstico e tratamento de pacientes com $\mathrm{CM}^{20,34,39}$. A distribuição de serviços de quimioterapia e radioterapia no âmbito dos SUS indica concentração nos grandes centros urbanos do país ${ }^{20,34}$. Além disso, em razão da concentração dos tratamentos oferecidos pelo SUS nas grandes cidades, muitos pacientes necessitam percorrer longas distâncias para terem acesso ao tratamento oncológico ${ }^{20,21,34}$.

As desigualdades na oferta de assistência especializada, para o diagnóstico e tratamento do $\mathrm{CM}$, refletem as diferenças no acesso ao serviço de saúde entre as Macrorregiōes do país ${ }^{20,40}$. Em 2010, 40\% do total de atendimentos realizados no âmbito do SUS foram concentrados em apenas sete capitais brasileiras, localizadas no Sul, Sudeste e Nordeste do Brasi ${ }^{34}$. Além disso, os maiores números de Cacon e Unacon habilitados no SUS estão localizados nessas Regiôes ${ }^{41}$. Essa realidade pode justificar a maior chance de atraso no tratamento para os residentes das Regiōes Centro-Oeste e Norte, onde são observados verdadeiros vazios sanitários ${ }^{34}$. No Sudeste do Brasil, onde há uma grande disponibilidade de serviços de quimioterapia e radioterapia, a maior probabilidade de atraso pode ser atribuída à alta demanda por atendimento, em virtude das altas taxas de incidência de CM nessa Região $0^{20,34,41}$. Residir na Regiāo Nordeste conferiu proteção para o atraso no início do tratamento. Achados recentes corroboram esse resultado ao evidenciarem que, nessa localidade, foram observados os menores intervalos de tempo, considerando as demais Regióes do Brasi $1^{1,41}$.

Neste estudo, foi observado que $54,9 \%$ da população não residiam no mesmo município da unidade de tratamento, e que os indivíduos que necessitavam se deslocar para outra localidade tiveram 16\% maior chance de atraso do que os que foram tratados no mesmo município de residência. Esse dado se assemelha ao de outro estudo brasileiro, no qual foi verificado que $51,34 \%$ dos pacientes, usuários do SUS, precisavam ir para outro município em busca de tratamento ${ }^{21}$. Evidencia-se, portanto, que as barreiras geográficas influenciam no acesso à saúde, o que já foi relatado no Estado de Minas Gerais no estudo de Alves et al. ${ }^{42}$. As desigualdades no acesso às açóes de rastreamento, diagnóstico e tratamento podem justificar o aumento nas taxas de mortalidade de mulheres que residem fora das capitais ${ }^{43}$. De acordo com Oliveira et al., ${ }^{34}$ a oferta de transporte e acomodação aos pacientes que necessitam podem ser estratégias para minimizar a barreira ocasionada pela distância entre o local de residência e o de tratamento.

Com relação à variável origem do encaminhamento, apesar da fragilidade desse dado por causa do grande percentual de falta de informação, observou-se que pacientes encaminhados pelo SUS apresentaram maiores chances de atraso do que aqueles que acessaram o serviço de saúde por meios próprios ou receberam outro tipo de encaminhamento. De acordo com Ferreira et al. ${ }^{44}$, que analisaram o tempo entre o diagnóstico e o início do tratamento do CM no Ceará, entre 2009 e 2011, os pacientes referenciados pelo SUS apresentaram maior tempo de espera do que aqueles provenientes de serviços privados, com intervalos de 71,5 dias e 39 dias $(\mathrm{p}=0,031)$, respectivamente. Medeiros et al. ${ }^{9}$ verificaram, por meio dos dados cadastrados no RHC, no período de 2000 a 2011, que pacientes encaminhadas pelo SUS apresentaram 1,34 vezes maior chance de atraso do que aquelas não encaminhadas pelo SUS. Usuárias do SUS também apresentam desvantagens com relação ao acesso ao diagnóstico e ao tratamento do $\mathrm{CM}^{9}$. Liedke et al. ${ }^{16}$ verificaram que as mulheres tratadas no SUS apresentam maior chance de serem diagnosticadas com EC avançado do que aquelas dos serviços privados. Esses autores afirmam que o acesso precoce ao diagnóstico e ao tratamento do CM poderia melhorar os desfechos das usuárias dos serviços públicos de saúde. Kaliks et al. ${ }^{45}$ e Oliveira et al.$^{34}$ relatam que a limitação estrutural do sistema de saúde público dificulta o acesso ao tratamento e, consequentemente, repercute negativamente no prognóstico.

A estrutura dos serviços de saúdes pode afetar significativamente o período de investigação diagnóstica e o tratamento de pacientes com $\mathrm{CM}^{46}$. Quando avaliada a unidade de tratamento, pacientes tratados em Cacon apresentaram $20 \%$ maior chance de atraso quando comparados aos que trataram em outras unidades. Os centros de maior complexidade, além de terem, por obrigatoriedade, que realizar o diagnóstico definitivo e tratar todos os tipos de câncer, também devem oferecer tratamento cirúrgico, quimioterapia e radioterapia. Essas atribuiçóes, em um único centro, podem estar gerando uma sobrecarga que estejam interferindo nesse resultado ${ }^{34,42}$. Por outro lado, as fragilidades no processo de regionalização da assistência podem favorecer a 
concentração do volume de atendimentos e procedimentos nas unidades de maior complexidade $21,34,42$.

Em estudos realizados em um Cacon localizado no Rio de Janeiro, foi observado que as pacientes encaminhadas sem diagnóstico, e que realizaram a biópsia no próprio Cacon, apresentaram maior chance de atraso para a confirmação diagnóstica ${ }^{36,47}$. Nesses casos, aguardar pelo diagnóstico em um hospital terciário especializado no tratamento do CM pode favorecer para que o período de espera pelo procedimento seja superior ao encontrado pelos pacientes que são diagnosticados em serviços de menor complexidade ${ }^{36}$.

$\mathrm{O}$ presente estudo apresenta algumas limitaçóes que devem ser pontuadas. Os dados deste estudo foram provenientes dos casos de câncer registrados no SisRHC do Brasil. A utilização de dados secundários, de caráter retrospectivo, pode interferir nos resultados observados. Entre os aspectos negativos, pontuam-se a grande quantidade de casos com variáveis náo preenchidas e a utilização de dados de origem secundária, cuja qualidade de coleta e armazenamento não podem ser garantidos. Como exemplo, destacam-se as variáveis raça/cor da pele, estado conjugal, consumo de álcool e tabaco, e origem do encaminhamento que, apesar de terem se mostrado significantes com relação ao desfecho estudado, apresentaram um percentual considerável de falta de informação, em grande parte, pelo fato de, no Estado de Sáo Paulo, esses dados náo serem rotineiramente coletados. Dessa forma, ressalta-se que é imprescindível o devido preenchimento dos dados do SisRHC, tendo em vista que sua análise pode auxiliar no monitoramento da assistência prestada aos pacientes oncológicos e no direcionamento de políticas públicas.

O tamanho amostral robusto pode ter favorecido possíveis erros nos testes de hipóteses, que podem ser caracterizados pela elevada frequência de associaçóes estatisticamente significantes, intervalos de confiança estreitos, mesmo quando as associaçóes entre a exposição e o desfecho eram fracas. Esse fator contribuiu para a decisão de não realizar análise ajustada, visto que praticamente todas as variáveis seriam inseridas na análise de regressão múltipla e, de maneira enviesada, permaneceriam no modelo final.

Como aspecto positivo, destaca-se a boa validade externa deste estudo. Por contemplar dados de todos os Estados do Brasil, considera-se que os resultados aqui apresentados podem ser extrapolados a pacientes diagnosticados com CM em todo o território nacional. Além disso, a heterogeneidade da população aqui estudada permite a comparabilidade dos dados a populaçóes de países com perfil semelhante ao do Brasil. Como ponto forte do estudo, também se pode salientar a comparação dos períodos antes e após a publicação da "Lei dos 60 dias", que demonstra que, mesmo após a fixação do prazo para o início do tratamento oncológico ter sido estabelecida legalmente, a maior parte da população com CM não está sendo beneficiada com essa medida. E ainda mais preocupante é que as chances de atraso aumentaram após a disposiçáo da Lei. Por outro lado, deve-se considerar que o período de cinco anos, após a publicação da Lei, pode náo ter sido suficiente para mudar o panorama de acesso ao tratamento oncológico no Brasil, visto que isso envolve um processo complexo de reorganização e adequação da rede pública à nova determinação do Ministério da Saúde. Novos estudos devem ser realizados futuramente para avaliação do impacto da Lei ao longo do tempo.

\section{CONCLUSÃO}

Foi verificado que a mediana do intervalo de tempo entre o diagnóstico e o início do primeiro tratamento oncológico de pacientes com CM cadastrados no SisRHC, diagnosticados entre 2000 e 2017, foi de 63 dias. Na maioria dos casos, houve atraso maior do que 60 dias $(52,8 \%)$. Fatores sociodemográficos, clínicos e relacionados ao tratamento influenciaram nesse atraso.

A partir da identificação do perfil de pacientes mais vulneráveis ao atraso, é possível contribuir com ações direcionadas a esses grupos específicos. Além disso, foi identificado que, mesmo após o estabelecimento da "Lei dos 60 dias", esse atraso continua sendo observado, sugerindo que devem ser priorizadas a reorganização e a fiscalização da atenção aos pacientes com CM.

\section{CONTRIBUIÇÕES}

Anke Bergmann, Luiz Claudio Santos Thuler, Giselle Coutinho de Medeiros, Clarice Gomes Chagas Teodózio, Erica Alves Nogueira Fabro e Suzana Sales de Aguiar contribuíram na concepçáo e planejamento do estudo, bem como na obtenção, análise e interpretação dos dados, e na redação do manuscrito. Artur Henrique Machado Lopes, Bárbara Cordeiro de Conte, Erisvan Vieira da Silva, Lyssandra Luiza Pestana Coelho, Nitza Ferreira Muniz e Sara Isabel Pimentel de Carvalho Schuab contribuíram na análise e interpretação dos dados e na redaçáo do manuscrito. Todos os autores aprovaram a versão final a ser publicada.

\section{DECLARAÇÃO DE CONFLITO DE INTERESSES}

A autora Anke Bergmann declara potencial conflito de interesses pela condição de ser a editora científica da Revista Brasileira de Cancerologia do INCA. Os demais autores não possuem conflito de interesses. 


\section{FONTES DE FINANCIAMENTO}

Não há.

\section{REFERÊNCIAS}

1. Instituto Nacional de Câncer José Alencar Gomes da Silva. Estimativa 2020: incidência de câncer no Brasil [Internet]. Rio de Janeiro: INCA; 2019 [acesso 2020 fev 08]. Disponível em: https://www.inca.gov.br/sites/ ufu.sti.inca.local/files/media/document/estimativa-2020incidencia-de-cancer-no-brasil.pdf.

2. Ferlay J, Colombet M, Soerjomataram I, et al. Estimating the global cancer incidence and mortality in 2018: GLOBOCAN sources and methods. Int J Cancer. 2019;144(8):1941-53. doi: https://doi.org/10.1002/ ijc.31937

3. Instituto Nacional de Câncer José Alencar Gomes da Silva. Atlas On-line de Mortalidade [Internet]. Rio de Janeiro: INCA; c1996-2014 - [atualizado 2019 maio 30; acesso 2020 fev 7]. Disponível em: http://mortalidade. inca.gov.br/Mortalidade/preparar Modelo05.action

4. Allemani C, Matsuda T, Di Carlo V, et al. Global surveillance of trends in cancer survival 2000-14 (CONCORD-3): analysis of individual records for 37513025 patients diagnosed with one of 18 cancers from 322 population-based registries in 71 countries. Lancet. 2018;391(10125):1023-75. doi: https://doi. org/10.1016/S0140-6736(17)33326-3

5. Thuler LCS, Bergmann A. Male breast cancer: clinicalepidemiological characteristics of 1189 Brazilian patients. Aging Male. 2015;18(2):118-23. doi: https://doi.org/10 $.3109 / 13685538.2014 .922532$

6. Bender PFM, Oliveira LL, Costa CR, et al. Men and women show similar survival rates after breast cancer. J Cancer Res Clin Oncol. 2017;143(4):563-71. doi: https://doi.org/10.1007/s00432-016-2311-4

7. Unger-Saldaña K. Challenges to the early diagnosis and treatment of breast cancer in developing countries. World J Clin Oncol. 2014;5(3):465-77. doi: https://doi. org/10.5306/wjco.v5.i3.465

8. Ghoncheh M, Pournamdar Z, Salehiniya H. Incidence and mortality and epidemiology of breast cancer in the world. Asian Pac J Cancer Prev. 2016;17(S3):43-6. doi: https://doi.org/10.7314/apjcp.2016.17.s3.43

9. Medeiros GC, Bergmann A, Aguiar SS, et al. Análise dos determinantes que influenciam o tempo para o início do tratamento de mulheres com câncer de mama no Brasil. Cad Saúde Pública. 2015;31(6):1269-82. doi: https:// doi.org/10.1590/0102-311X00048514

10. Freitas AGQ, Weller M. Patient delays and system delays in breast cancer treatment in developed and developing countries. Cienc Saúde Colet. 2015;20(10):3177-89. doi: https://doi.org/10.1590/1413-812320152010.19692014
11. Caplan L. Delay in breast cancer: implications for stage at diagnosis and survival. Front Public Health. $2014 \mathrm{Jul}$ 29;2:87. doi: https://doi.org/10.3389/fpubh.2014.00087

12. Williams F. Assessment of breast cancer treatment delay impact on prognosis and survival: a look at the evidence from systematic analysis of the literature. J Cancer Biol Res. 2015;3(4):1071.

13. Ministério da Saúde (BR), Gabinete do Ministro. Portaria No. 876, de 16 de maio de 2013. Dispóe sobre a aplicaçăo da Lei $\mathrm{n}^{\circ} 12.732$, de 22 de novembro de 2012, que versa a respeito do primeiro tratamento do paciente com neoplasia maligna comprovada, no âmbito do Sistema Único de Saúde (SUS). Diário Oficial da União, Brasília, DF; 2013 maio 17. Seção I, p. 135.

14. Presidência da República (BR). Lei no 12.732, de 22 de novembro de 2012. Dispóe sobre o primeiro tratamento de paciente com neoplasia maligna comprovada e estabelece prazo para seu início. Diário Oficial da União, Brasília, DF; 2012 nov 23. Seção I, p. 1.

15. Lee BL, Liedke PE, Barrios $\mathrm{CH}$, et al. Breast cancer in Brazil: present status and future goals. Lancet Oncol. 2012;13(3):e95-e102. doi: https://doi.org/10.1016/ S1470-2045(11)70323-0

16. Liedke PE, Finkelstein DM, Szymonifka J, et al. Outcomes of breast cancer in Brazil related to health care coverage: a retrospective cohort study. Cancer Epidemiol Biomarkers Prev. 2014;23(1):126-33. doi: https://doi. org/10.1158/1055-9965.EPI-13-0693

17. Sousa SMMT, Carvalho MGFM, Santos Junior LA, et al. Acesso ao tratamento da mulher com câncer de mama. Saúde Debate. 2019;43(122):727-41. doi: https://doi. org/10.1590/0103-1104201912206

18. Cabral ALLV, Giatti L, Casale C, et al. Vulnerabilidade social e câncer de mama: diferenciais no intervalo entre o diagnóstico e o tratamento em mulheres de diferentes perfis sociodemográficos. Cienc Saúde Colet. 2019;24(2):613-22. doi: https://doi.org/10.1590/141381232018242.31672016

19. Romeiro Lopes TC, Gravena AAF, Demitto MO, et al. Delay in diagnosis and treatment of breast cancer among women attending a reference service in Brazil. Asian Pac J Cancer Prev. 2017;18(11):3017-23. doi: https://doi. org/10.22034/APJCP.2017.18.11.3017

20. Silva GA, Bustamante-Teixeira MT, Aquino EML, et al. Acesso à detecçáo precoce do câncer de mama no Sistema Único de Saúde: uma análise a partir dos dados do Sistema de Informaçóes em Saúde. Cad Saúde Pública. 2014;30(7):1537-50. doi: https://doi. org/10.1590/0102-311X00156513

21. Saldanha RF, Xavier DR, Carnavalli KM, et al. Estudo de análise de rede do fluxo de pacientes de câncer de mama no Brasil entre 2014 e 2016. Cad Saúde Pública. 2019;35(7):e00090918. doi: https://doi. org/10.1590/0102-311x00090918 
22. Instituto Nacional de Câncer. Registros hospitalares de câncer: planejamento e gestão. 2. ed. Rio de Janeiro: INCA; 2010.

23. Programa das Naçóes Unidas para o Desenvolvimento (PNUD) [Internet]. Brasília, DF: PNUD; c2020. Ranking IDHM Municípios 2010; [2013] [acesso 2020 jun 5]. Disponível em: https://www.br.undp. org/content/brazil/pt/home/idh0/rankings/idhmmunicipios-2010.html

24. Tribunal de Contas da União (BR). Política Nacional de Atenção Oncológica. Brasília, DF: TCU, Secretaria de Fiscalização e Avaliação de Programas de Governo; 2011. (Relatório de auditoria operacional).

25. Ministério da Saúde (BR). Portaria No. 140, de 27 de fevereiro de 2014. Redefine os critérios e parâmetros para organização, planejamento, monitoramento, controle e avaliação dos estabelecimentos de saúde habilitados na atenção especializada em oncologia e define as condições estruturais, de funcionamento e de recursos humanos para a habilitação destes estabelecimentos no âmbito do Sistema Único de Saúde (SUS). Diário Oficial da União, Brasília, DF; 2014 abr 2. Seção I, p. 60.

26. McLaughlin JM, Anderson RT, Ferketich AK, et al. Effect on survival of longer intervals between confirmed diagnosis and treatment initiation among low-income women with breast cancer. J Clin Oncol. 2012;30(36):4493-4500. doi: https://doi.org/10.1200/ JCO.2012.39.7695

27. Mujar M, Dahlui M, Yip CH, et al. Delays in time to primary treatment after a diagnosis of breast cancer: does it impact survival? Prev Med. 2013;56(3-4):222-4. doi: https://doi.org/10.1016/j.ypmed.2012.12.001. Erratum in: Prev Med. 2014 Apr;61:128.

28. Yoo TK, Han W, Moon HG, et al. Delay of treatment initiation does not adversely affect survival outcome in breast cancer. Cancer Res Treat. 2016 Jul;48(3):962-9. doi: https://doi.org/10.4143/crt.2015.173

29. Travassos C, Bahia L. Qual é a agenda para o combate à discriminação no SUS? Cad Saúde Pública. 2011;27(2):204-5. doi: https://doi.org/10.1590/S0102311X2011000200001

30. Rayne S, Schnippel K, Thomson J, et al. Male breast cancer has limited effect on survivor's perceptions of their own masculinity: a record review and telephone survey of patients in Johannesburg, South Africa. Am J Men's Health. 2016;11(2):246-52. doi: https://doi. org/10.1177/1557988316631512

31. Mattarella A. Breast cancer in men. Radiol Technol. 2010;81(4):361M-378M.

32. Leon-Ferre RA, Giridhar KV, Hieken TJ, et al. A contemporary review of male breast cancer: current evidence and unanswered questions. Cancer Metastasis Rev. 2018;37(4):599-614. doi: https://doi.org/10.1007/ s10555-018-9761-x
33. Howlader N, Noone A, Krapcho MC, et al., editors. SEER Cancer statistics review, 1975- 2014 [Internet]. Bethesda, MD: National Cancer Institute; 2017. [updated 2018 Apr 2; cited 2020 Feb 12]. Available from: https://seer.cancer.gov/archive/csr/1975_2014/

34. Oliveira EXG, Melo EC, Pinheiro RS, et al. Acesso à assistência oncológica: mapeamento dos fluxos origem-destino das internaçóes e dos atendimentos ambulatoriais. O caso do câncer de mama. Cad Saúde Pública. 2011;27(2):317-26. doi: https://doi. org/10.1590/S0102-311X2011000200013

35. Marmot M. Social determinants of health inequalities. Lancet. 2005;365(9464):1099-1104. doi: https://doi. org/10.1016/S0140-6736(05)71146-6

36. Medeiros GC, Thuler LCS, Bergmann A. Delay in breast cancer diagnosis: a Brazilian cohort study. Public Health. 2019 Feb;167:88-95. doi: https://doi.org/10.1016/j. puhe.2018.10.012

37. Höfelmann DA, Anjos JC, Ayala AL. Sobrevida em dez anos e fatores prognósticos em mulheres com câncer de mama em Joinville, Santa Catarina, Brasil. Cienc Saúde Colet. 2014;19(6):1813-24. doi: https://doi. org/10.1590/1413-81232014196.03062013

38. Haddad NC, Carvalho ACA, Novaes CO. Perfil sociodemográfico e de saúde de mulheres submetidas à cirurgia para câncer de mama. Rev HUPE. 2015;14(Supl 1):28-35. doi: https://doi.org/10.12957/ rhupe.2015.17923

39. Oliveira EXG, Pinheiro RS, Melo ECP, et al. Condicionantes socioeconômicos e geográficos do acesso à mamografia no Brasil, 2003-2008. Cienc Saúde Colet. 2011;16(9):3649-64. doi: https://doi.org/10.1590/ S1413-81232011001000002

40. Ministério da Saúde (BR), Secretaria de Vigilância em Saúde, Departamento de Análise de Situação em Saúde. Saúde Brasil 2008: 20 anos de Sistema Único de Saúde (SUS) no Brasil. Brasília, DF: Ministério da Saúde; 2009. (Série G. Estatística e Informação em Saúde). Capítulo 6, Tendências e controle do câncer e os 20 anos de Sistema Único de Saúde no Brasil; p. 365-84.

41. Instituto Nacional de Câncer José Alencar Gomes da Silva. A situação do câncer de mama no Brasil: síntese de dados dos sistemas de informação. Rio de Janeiro: INCA; 2019.

42. Alves MO, Magalhães SCM, Coelho BA. A regionalização da saúde e a assistência aos usuários com câncer de mama. Saúde Soc. 2017;26(1):141-154. doi: https:// doi.org/10.1590/S0104-12902017160663

43. Silva GA. Câncer de mama no Brasil: estratégias para o seu enfrentamento. Cad Saúde Pública. 2012;28(1):4-5. doi: https://doi.org/10.1590/S0102-311X2012000100001

44. Ferreira NAS, Carvalho SMF, Valenti VE, et al. Treatment delays among women with breast cancer in a low socioeconomic status region in Brazil. BMC Womens Health. 
2017;17(1):13. doi: https://doi.org/10.1186/s12905016-0359-6

45. Kaliks RA, Pontes LB, Bognar CLFB, et al. Pacientes com câncer de mama oriundas do Sistema Único de Saúde tratadas no setor privado: custos de um piloto de parceria público-privada em oncologia. Einstein (São Paulo). 2013;11(2):216-23. doi: https://doi.org/10.1590/ S1679-45082013000200014

46. Gonçalves LLC, Travassos GL, Almeida AM, et al. Barreiras na atenção em saúde ao câncer de mama: percepção de mulheres. Rev Esc Enferm USP. 2014;48(3):394-400. doi: https://doi.org/10.1590/ S0080-623420140000300002

47. Rezende MCR, Koch HA, Figueiredo JA, et al. Causas do retardo na confirmação diagnóstica de lesões mamárias em mulheres atendidas em um centro de referência do Sistema Único de Saúde no Rio de Janeiro. Rev Bras Ginecol Obstet. 2009;31(2):75-81. doi: https://doi. org/10.1590/S0100-72032009000200005 